\title{
Tuning light transmission with smart fluids based on 1D carbon nanomaterials
}

\author{
A. P. Franco-Bacca ${ }^{1,2}$, F. Cervantes-Alvarez ${ }^{1}$, C. Glorieux ${ }^{2}$, \\ G. Rodríguez-Gattorno ${ }^{1}$ and J. J. Alvarado-Gil ${ }^{1,2}$. \\ ${ }^{1}$ Applied Physics Department, Cinvestav-Unidad Mérida, Carretera Antigua a \\ Progreso Km. 6, Mérida, Yucatán, Mexico, 97310, Mexico. \\ ${ }^{2}$ Laboratory of Soft Matter and Biophysics, Department of Physics and Astronomy, \\ KU Leuven, Celestijnenlaan 200D, 3001 Heverlee, Belgium.
}

\begin{abstract}
This work reports on a study of light transmission in electrically responsive materials consisting of long aspect ratio carbon nanostructures in fluid matrices. Composite fluids were prepared using multiwalled carbon nanotubes or carbon nanofibers in two types of fluid matrices, ethylene glycol and distilled water. Measurements were performed illuminating the sample with polarized light. Before, during and after applying a uniform and constant electric field, the evolution of the intensity of the transmitted light was monitored. For ethylene glycol-based dispersions, it was found that the applied electric field induces substantial changes in the polarization component of the incident light parallel with the electric field vector. In contrast, for the same dispersions, the optical transmission of the light polarized in the direction perpendicular to the electric field does not show appreciable changes. The matrix fluid properties were found to be crucial for the electro-optical effect: in distilled waterbased dispersions, no changes of transmission were found upon applying an electric field for any of the polarization components of the light. It was also shown that the polarization of the transmitted light can be dynamically adjusted in time and space by varying the applied voltage across ethylene glycol dispersions. The electric control of the light polarization opens the possibility of using these simple systems as light dimmers or in a more sophisticated application such as reversible dynamic polarizers.
\end{abstract}

\section{Introduction}

In recent years a high number of nanomaterials with a diversity of physical properties have been developed, enabling a technological revolution in materials science and its applications [1,2]. Since their discovery, carbon nanostructures have become a class of highly promising materials due to their outstanding mechanical, electrical, optical and thermal properties $[3,4]$. It has been shown that those carbon nanostructures can serve as a useful basis for the development of novel composites[5,6]. In particular, when the composites are present in the form of dispersions of particles in fluid matrices, they can act as composites with response to external stimuli, laying a basis for smart fluids[7-10]. These phenomena are based on the fact that, when applying an AC electric field, carbon nanotubes and nanofibers can become aligned along the direction of the field[7,11-14]. The response of carbon nano-inclusions to the application of both 
DC and AC electric fields has been found to be useful orienting and purifying CNTs $[15,16]$ in liquid matrices. In the case of liquids that can be polymerized after orienting the particles, it has been shown that the induced order is useful in enhancing the mechanical, electrical and thermal properties in the final solid composite [4,17-20]. In the case of fluid matrices, it has also been shown that the application of electric fields to these fluids allows that their electrical, mechanical and thermal properties can be changed in a controlled fashion at will and in a reversible way[4,9,11]. One important aspect that has been reported in the literature is that the application of AC electric fields presents better results in the alignment and structure formation of the carbon nanoinclusions[16,17,21]. Additionally, it is also expected that the electric field induced nanostructuring of the fluid composite shows light polarization dependence.

Even though a great variety of composite fluids based on this kind of dispersions has been developed, it must be taken into account that each new association of particles and fluids involves a new defy as well as new phenomena and possibilities. One of the most significant challenges is to avoid to a maximum extent the agglomeration of the particles in order to prevent that these very large structures precipitate by sedimentation. Several approaches have shown successful results at least in time scales adequate for a variety of possible practical applications. By appropriately choosing the liquid matrix and the adequate surfactant, it is possible to delay the natural agglomerative behavior of the particles as well as their eventual sedimentation. In contrast, when an electric field is applied, although the carbon nanostructures become intentionally agglomerated, the presence of the field allows to keep the structure formed in a fixed configuration [22,23].

The interaction of polarized light with arrangements of carbon nanoparticles has been studied by several research groups. Brown et al.[12] reported the alignment dependence of single-wall carbon nanotubes on the electric field using polarimetry. Shoji et al.[24] developed a uniaxially aligned polarizer, using mechanical stretching of a material containing carbon nanotubes embedded in a polymer film. Kang et al.[25] studied the light absorption of a single carbon nanotubes cluster, stretched by an electric field when the polarization vector of the incident light is parallel to the applied electric field. Lynch and Patrick [26] reported the alignment of carbon nanotubes using a liquid crystal matrix, by applying a magnetic or electric field. Additionally, Jeong et al.[27] reported the electroactive elongation of a carbon nanotube aggregate, through the application of an electric field in a liquid crystal medium. The magnitude of the changes in the light transmission as well as the involved time scales, and its relationship with the complex microscopic structuring are defining phenomena, which determine the possible application of these composites and therefore, they are subjects that deserve a more extensive investigation. [28]

In this work, we report the analysis of the light transmission through dispersions of multiwall carbon nanotubes and carbon nanofibers in ethylene glycol or water, aligned when an AC electric field is applied. Different electric field values and particle concentrations were tested. It is also shown that the polarizing effect of the chains nanoparticles structure, generated by the electric field, opens the possibility of using these simple systems as light dimmers or maybe in a more sophisticated application as reversible dynamic polarizers. The redispersion of nanoparticles resets the system, leaving it ready to adjust its light transmission in a wide range of values with the application of a determined electric field value.

\section{Experimental Details}




\subsection{Sample preparation}

The samples were prepared in two types of fluid matrices: ethylene glycol (SigmaAldrich, 99\%) and distilled water. The inclusions used were multiwall carbon nanotubes (CNT), with outer diameters of 10-20 nm and lengths ranging from 10-30 $\mu \mathrm{m}$ (Nanostructured and Amorphous Materials Inc.) and carbon nanofibers (CNF) (Pyrograf Products, Inc. PR-19-XT-HHT), with outer diameters of 1150-180 nm and lengths ranging from $60-80 \mu \mathrm{m}$. These were dispersed in the respective matrices using an ultrasonic bath with a power of $160 \mathrm{~W}$ with an operation frequency of $45 \mathrm{kHz}$. The samples were sonicated for six non-continuous periods of one hour.

Four different samples with $0.03,0.05,0.075$ and $0.1 \%$ weight fraction concentrations (\% wf, which is defined as the ratio of carbon nanomaterials to matrices weights) were prepared from both kinds of carbon nanomaterials in each dispersion media. In the case of distilled water dispersions, only the samples with 0.05 and $0.1 \%$ wf provided reliable experimental results as will be explained in section 3.1.

\subsection{Experimental setup}

In order to perform the optical experiments, the samples were placed inside a glass cell of $20 \mathrm{~mm}$ height, $7 \mathrm{~mm}$ wide and $1 \mathrm{~mm}$ thick. An aliquot of $50 \mu \mathrm{L}$ was taken for each measurement. The electric field was applied using two flat copper electrodes separated by a distance of $7 \mathrm{~mm}$.

Figure 1 shows a schematic diagram of the experimental setup that was used for the optical measurements. The beam of a diode laser $(100 \mathrm{~mW}, \lambda=633 \mathrm{~nm})$, modulated at $1 \mathrm{~Hz}$, was incident on the sample contained in the glass cell. The incident laser beam was linearly polarized by means of a rotating polarizer, either parallel or perpendicular with respect to the applied electric field. The transmitted light through the sample was monitored with a photodiode (Thorlabs DET36A/M), and a lock-in amplifier (Stanford Research Systems SR-830 DSP) connected to a PC via a GPIB interface. The CNT and $\mathrm{CNF}$ dispersed in ethylene glycol were subjected to three different voltages; $70 \mathrm{~V}_{\mathrm{rms}}$, $100 V_{r m s}$, and $130 V_{r m s}$, generating an electric field of $100 V_{r m s} / \mathrm{cm}, 143 V_{r m s} / \mathrm{cm}$, and $186 V_{r m s} / \mathrm{cm}$ respectively. Different voltages were applied with an AC Variac using the line voltage $(230 \mathrm{~V}$ at $50 \mathrm{~Hz})$. The transmitted light was monitored for $120 \mathrm{~s}$ before the voltage was applied and for $800 \mathrm{~s}$ with the voltage applied.

Based on the observation that after $300 \mathrm{~s}$ of applying the electric field to the sample, the transmittance remains constant, additional experiments were carried out to determine the dependence of the optical transmission on the polarization angle of the incident laser beam in such conditions. 


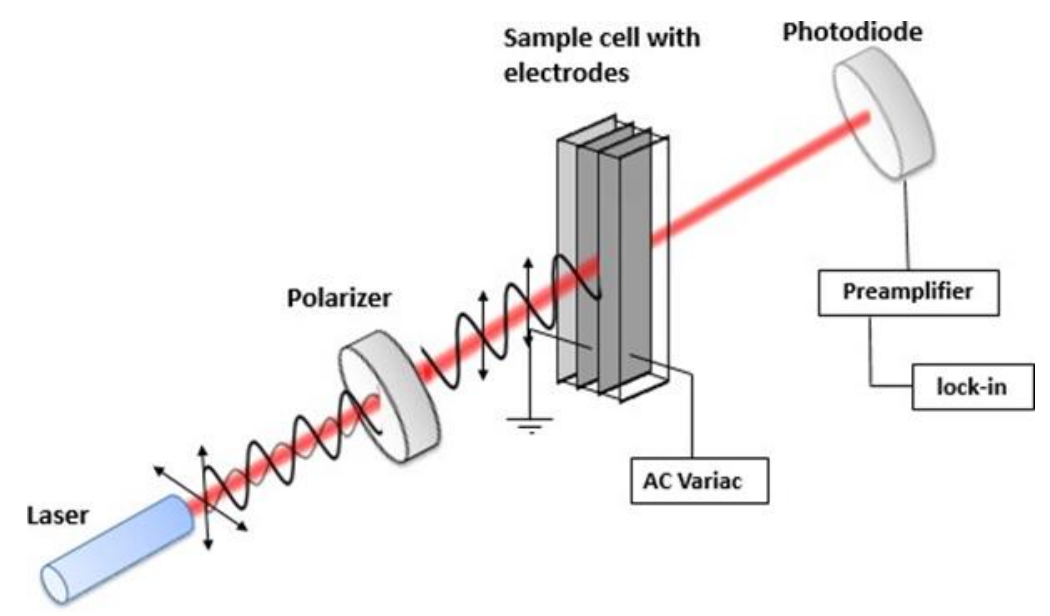

Figure 1 Schematic diagram of experimental setup used to determine the intensity of polarized light transmitted through the sample.

\section{Results and discussion}

\subsection{Optical images of the samples}

Figure 2 presents four pairs of micrographs that were taken before (left), and after (right) that a voltage of $100 \mathrm{~V}_{\text {rms }}$ was applied to the electrodes during 120s. Figures 2(a) and 2(b) show the image of CNF and CNT dispersed in distilled water with a concentration of $0.05 \%$ wf respectively. Figures 2(c) and 2(d) show the case of a sample of CNF and CNT dispersed in ethylene glycol with a concentration of $0.05 \%$ wf respectively. The scale bar in the lower right corner corresponds to $500 \mu \mathrm{m}$. The micrographs illustrate how the electric field induced an assembling effect. A video of the rearrangement dynamics of the particles is available at (moviel link). At the very beginning of the process, after the electric field is turned on, the particles aligned and move preferentially in the direction of the applied electric field. After a while, the chains of nanostructures agglomerates concatenated along the applied electric field, developing a structured pattern which remains stable.

(a)

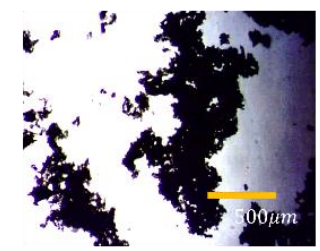

(c)

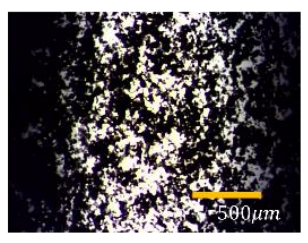

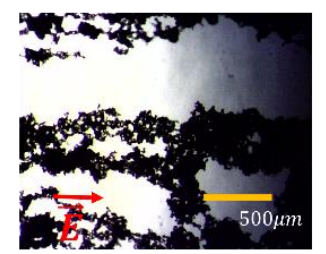

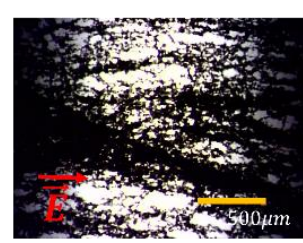

(b)

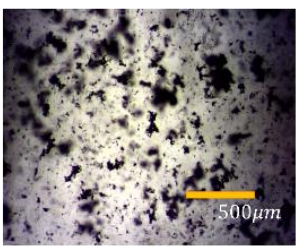

(d)

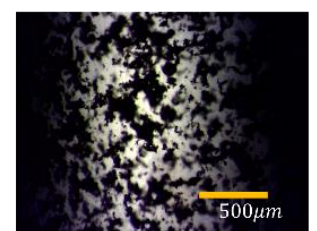

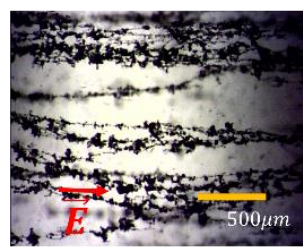

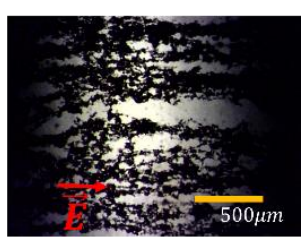

Figure 2 Optical micrographs of the samples with a) CNF 0.05\%wf b) CNT 0.05\%wf both dispersed in water and c) CNF $0.05 \%$ wf d) CNT 0.05\%wf both dispersed in ethylene glycol. 
The phenomenon of alignment of nano and microstructures of various geometries in fluid matrices has been the subject of several research works, which can be useful in understanding the behaviour of our fluid samples[29,30], and the determining role of dipole-dipole interaction. In our case, the behavior observed can be understood based on the fact that the carbon nanostructures are small agglomerations that are dispersed in a fluid without any specific order, as seen in figure 3(a). When the electric field is switched on, during the first stage of the process, the induced dipole-dipole interactions provoke the migration and new agglomerations of the carbon nanostructures, figure 3(b). Then there is a second stage where the agglomerations are aligned in parallel to the applied electric field as seen in figure 3(c).

(a)
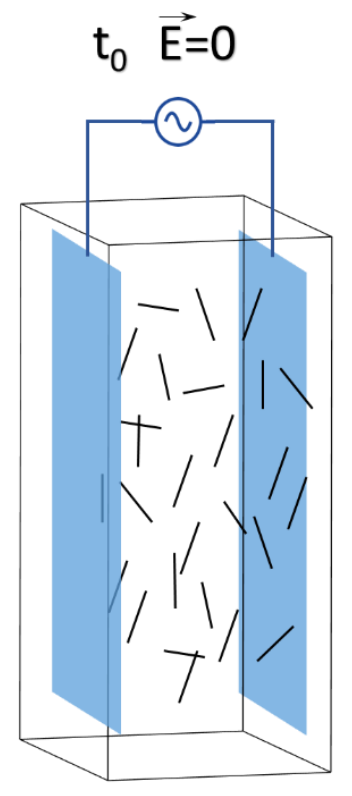

(b)
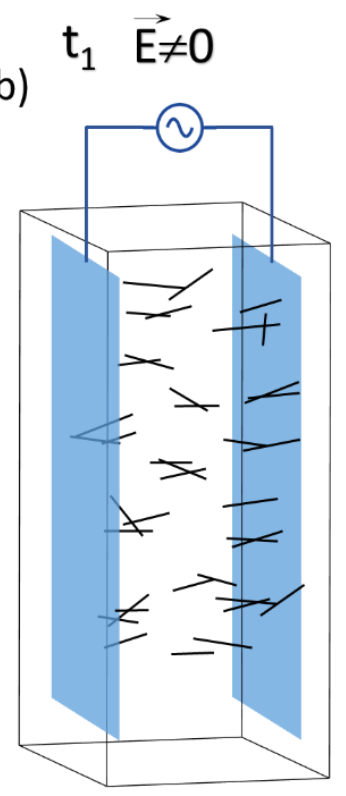

$t_{2} \quad \vec{E} \neq 0$

(c)

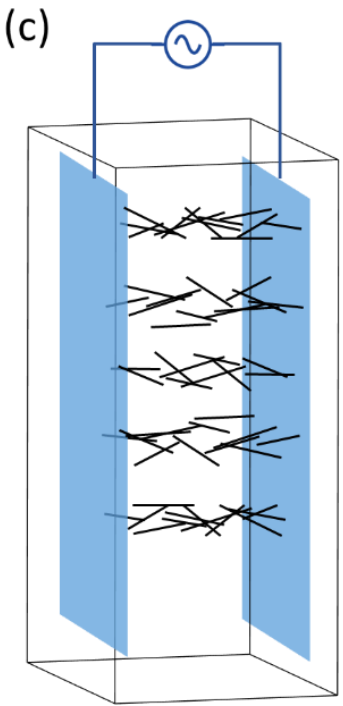

Figure 3 Diagram of the dispersed nanostructures in the fluid (a) without electric field. (b)First stage when the electric field is switched on. (c) Second stage where the agglomerations are aligned in parallel to the applied electric field.

Apparently, with increasing particle concentration, the migration effect leads to a fewer number of wider chain regions. In the case of lower particle concentrations, the initial distribution is more uniform, the agglomerates are smaller and the electric field inhomogeneities too. As a consequence, a stable morphology with thinner chains of small agglomerates is formed. Once agglomerates are residing in a low electric field chain region, they are immobilized and do not cross anymore the large motion stream to migrate to another nearby chain.

Some theoretical and experimental studies help to understand or explain why this phenomenon occurs. Shao-Jie et al.[31], from a quantum mechanics calculation, state that the rotational torque of $\mathrm{CNT}$ is proportional to the transversal and longitudinal polarizabilities and varies with respect of the angle between CNT and electric field.

Oliva-Avilés et al. [13] report the modeling of the dynamic of alignment and structure formation of CNT in viscous liquid matrices, under the application of AC electric field. Using the effective dipole modeling approach, they establish three mechanisms that 
govern the structure formation: CNT rotation and alignment, Coulombic interactions between CNT and migration of the CNT towards the electrodes.

From experimental and simulation work, Chen et al. [32] found that the high AC electric field gradient, produced between electrodes, is the force responsible for the alignment and interconnection, because of the dipolar moment induced in the CNT. The directional movement along the electric field gradient under an inhomogeneous electric field is referred to as dielectrophoresis (DEP) effect. They also simulated what happens when the CNT reaches an electrode and found that CNT became an electrode extension and that in the CNT apexes the AC electric field suffers important changes in its distribution increasing the electric field gradient, and consequently the DEP force, accelerating the interconnection process until electrodes get bridged.

\subsection{Polarized light transmission measurements}

Figure 4 shows the temporal evolution of the light intensity transmitted through the ethylene glycol dispersions with CNT (4a) and CNF (4b). The incident beam was perpendicularly polarized to the chains. The first 120 seconds elapsed without applying the electric field, in the next 800 seconds a voltage of $100 \mathrm{~V}_{\text {rms }}$ was applied. Note that the intensity variation during the first 120 seconds is quite small, indicating that no significant sedimentation of CNT occurs in the time scale of this experiment. As soon as the AC voltage is applied, a first stage is observed where the intensity of the transmitted light increases due to the formation of new glomerations. In the second stage these agglomerations form chains that resemble a mesh-like window. The intensity of light propagation decreases with increasing concentration of nanoparticles, both for nanotubes and nanofibers. Apparently, the effect of the increment in the number of absorbing entities in the total optical transmission, according to Beer's law[25], is stronger than the dependence on the light transmission corridors, between the chains of agglomerates, which were formed when the voltage was applied. The time needed for the stabilization of the chains also depends on the concentration, the higher the concentration of the particles, the faster the structure formation. In the case of CNF with $0.03 \%$ wf (Figure $3 \mathrm{~b}$ black line) the formation of the mesh window is slower compared to that of higher concentrations and when it reaches the maximum, which is difficult to determine, the transmitted light does not stabilize because some particles agglomerates remain isolated and they keep moving without forming a stable concatenated structure. 

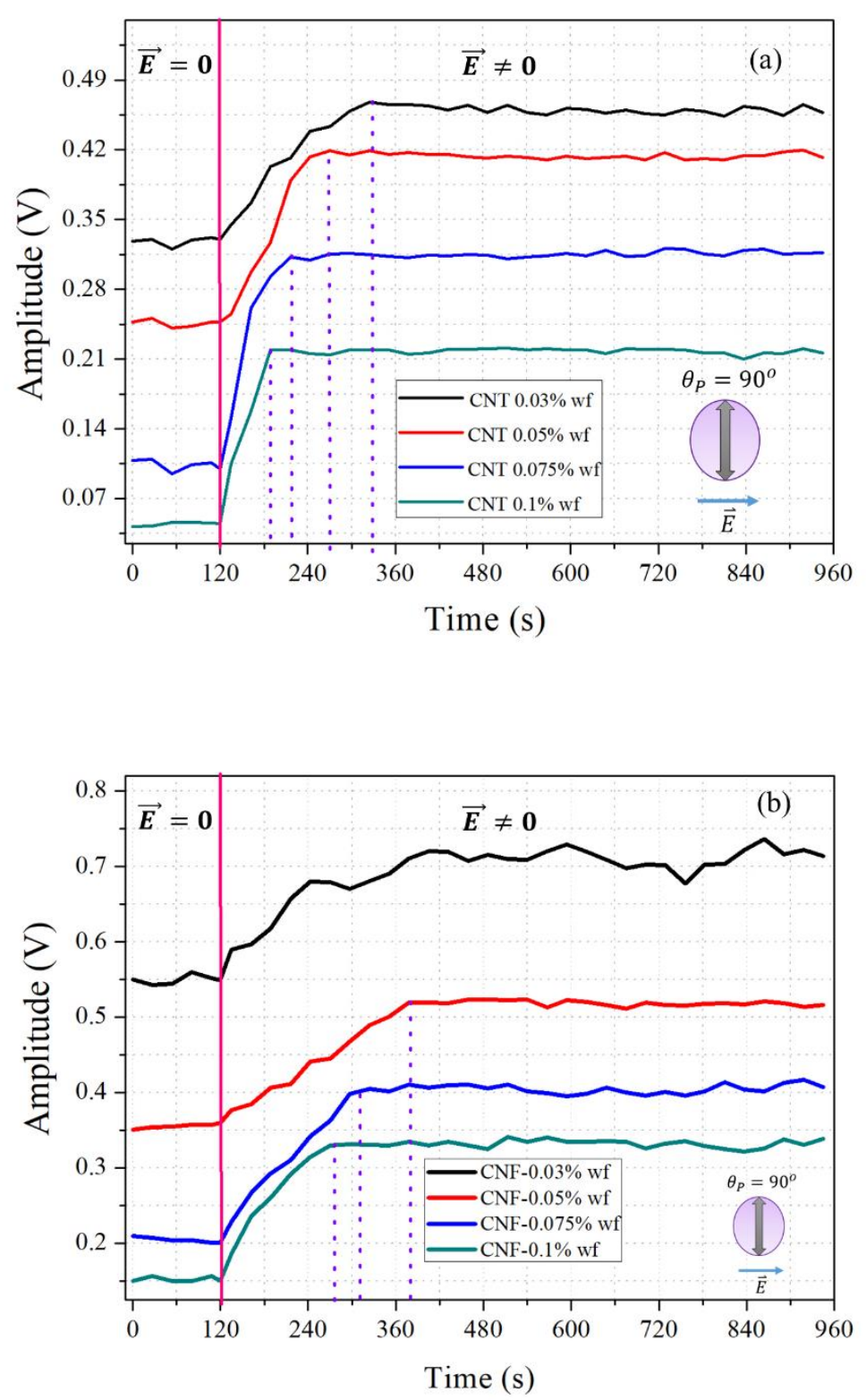

Figure 4 Transmitted light intensity evolution of polarized light, perpendicular to the chains structure, through dispersions of CNT (a) and CNF (b) in ethylene glycol. Four different concentrations were tested: $0.03 \% \mathrm{wf}$ (black), $0.05 \% \mathrm{wf}$ (red), $0.075 \% \mathrm{wf}$ (blue) and $0.1 \% \mathrm{wf}$ (green), during 120 seconds before switching on an AC voltage of $100 V_{r m s}$ and 800 seconds with applied field.

Figure 5 shows the time that it takes to form the mesh windows when applying three different voltages ( $70 \mathrm{~V}_{\mathrm{rms}}, 100 \mathrm{~V}_{\mathrm{rms}}$ and $130 \mathrm{~V}_{\mathrm{rms}}$ ) for ethylene glycol dispersions of CNT (solid symbols) and CNF (open symbols). It was estimated from the moment that the voltage is applied until the instant when the amplitude of transmitted light reaches its maximum value, as is indicated in Figure 4 (purple dotted lines). This time depends directly on the $\%$ wf concentration of particles and the magnitude of the applied voltage. The mesh window formation time decreases as the \% wf concentration and the magnitude of the applied voltage increase. The observed trends are intuitively expected since the agglomeration rate (and further alignment along the electric field) is predictable 
to be proportional to the initial concentration [33]. The dependence with respect to the applied voltage is related to the proportionality between the torque experienced by the dipole of the particle and the electric field [34]. In the dispersions with CNT, the mesh window formation time was always less than in those of CNF under the same conditions, which is probably related to its biggest dimensions. The CNF dispersion with $0.03 \%$ wf concentration was not stable at any of the three applied voltages, which made it impossible to determine the maximum amplitude value. The mesh window formation, in the CNT sample with a concentration of $0.1 \% \mathrm{wf}$ and $130 \mathrm{~V}_{\mathrm{rms}}$ applied, did not happen since the heating generated by the Joule effect, present in all measurements, increased the temperature up to the boiling point of the ethylene glycol.

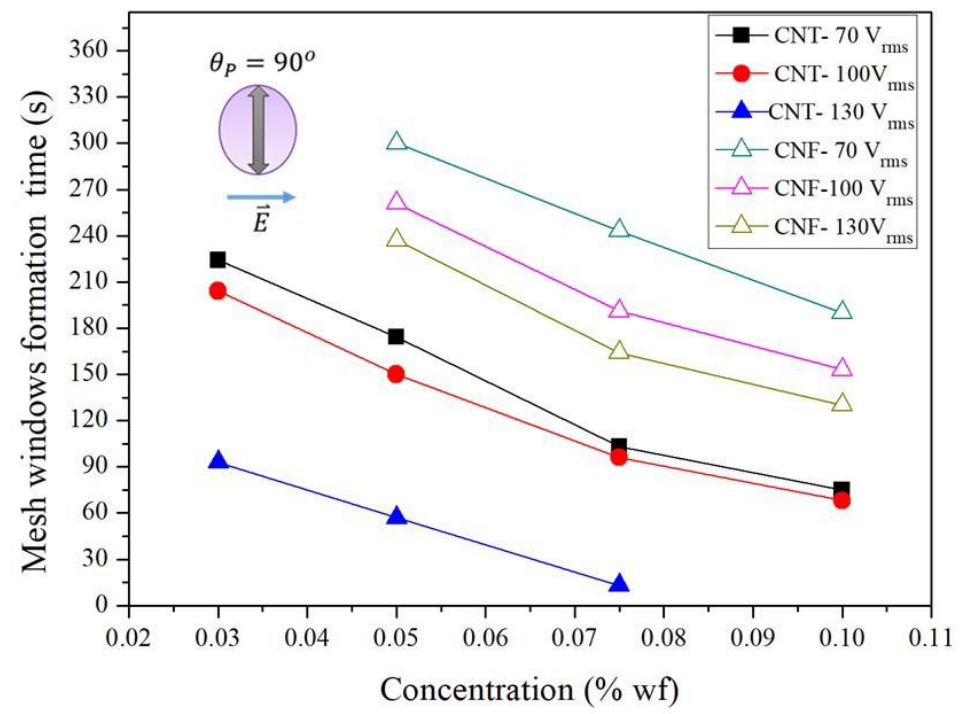

Figure 5 Mesh windows formation time from four different concentrations of CNT (black, red and blue solid symbol) and CNF (green, pink and brown, clear symbol) dispersions in ethylene glycol, when voltages of $70 V_{\text {rms }}$ (black and green), $100 V_{\text {rms }}$ (red and pink) and $130 V_{\text {rms }}$ (blue and brown) were applied. 

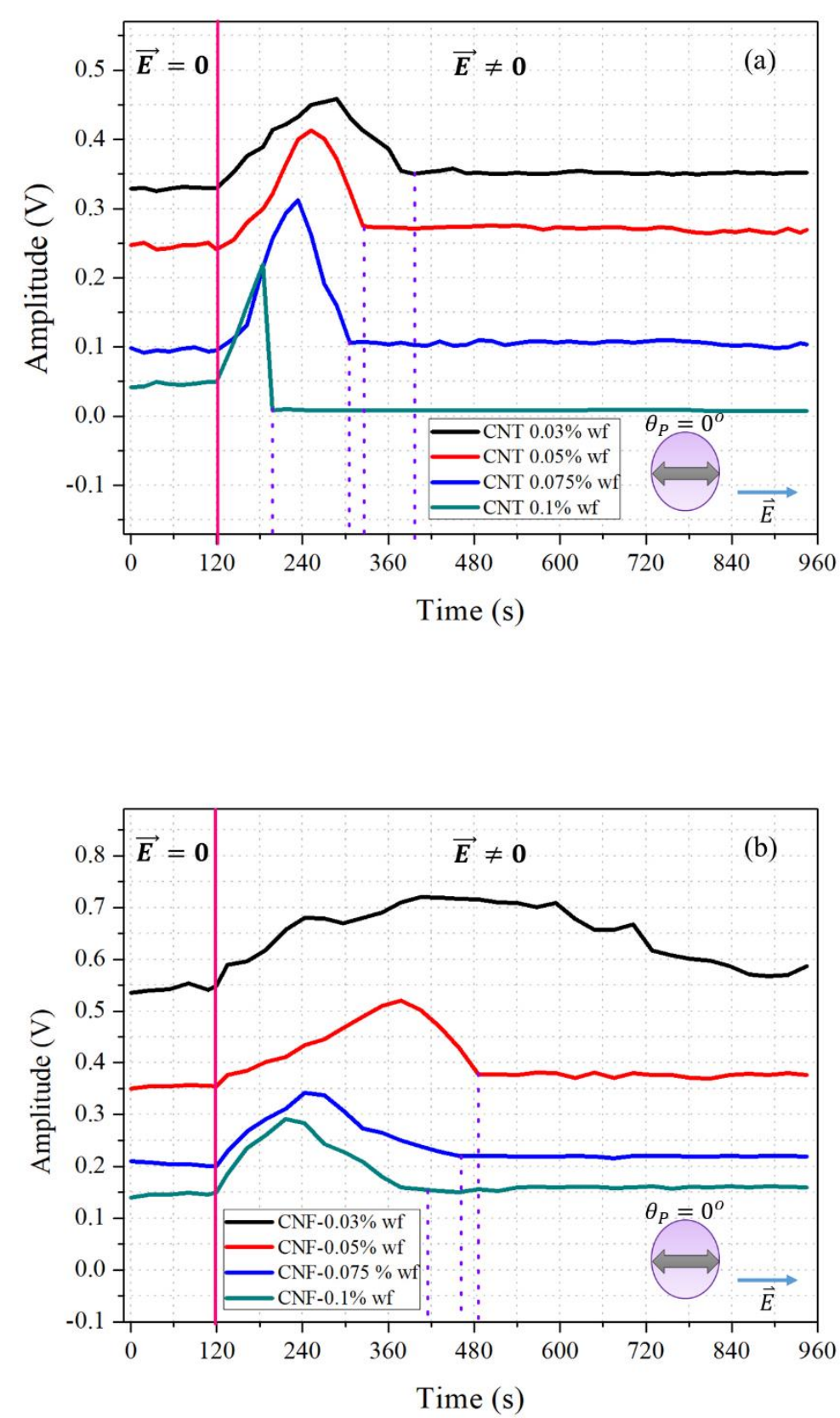

Figure 6. Transmitted light intensity evolution of polarized light, parallel to the chains structure, through dispersions of CNT (a) and CNF (b) in ethylene glycol. Four different concentrations were tested: $0.03 \% \mathrm{wf}$ (black), $0.05 \% \mathrm{wf}$ (red), $0.075 \% \mathrm{wf}$ (blue) and $0.1 \% \mathrm{wf}$ (green), during 120 seconds before switching on an AC voltage of $100 V_{r m s}$ and 800 seconds with applied field

Figures 6 shows the temporal dependence of the light intensity transmitted through ethylene glycol dispersions of $\mathrm{CNT}(6 \mathrm{a})$ and $\mathrm{CNF}(6 \mathrm{~b})$. Here the light was polarized parallel to the chains. As in the previous case, the first 120 seconds elapsed without applying the electric field, and in the next 800 seconds, a voltage of $100 \mathrm{~V}_{\text {rms }}$ was applied. When the electric field is turned on, the formation of the mesh window is evidenced in the same way that happens when the light is perpendicularly polarized to the chains. Nevertheless, after arriving at the maximum amplitude, it is observed how the amplitude is going down until it gets stable. This behavior depends on the $\%$ wf concentration of 
particles, and it is related to a polarizing effect that the chains structure has on the light that impinges the sample. Since this effect is not observed for pure ethylene glycol, it can be assumed that the applied electric field does not have an appreciable influence on the light transmitted. Therefore, the polarization dependence should be attributed to the influence that the electric field has on the induced anisotropy of the agglomeration morphology, and this in turn on the propagation of the light that is passing through the chain regions. It was observed that electrically conductive chains act as shortcuts for the electric field component of electromagnetic waves, which reduces optical transmission.

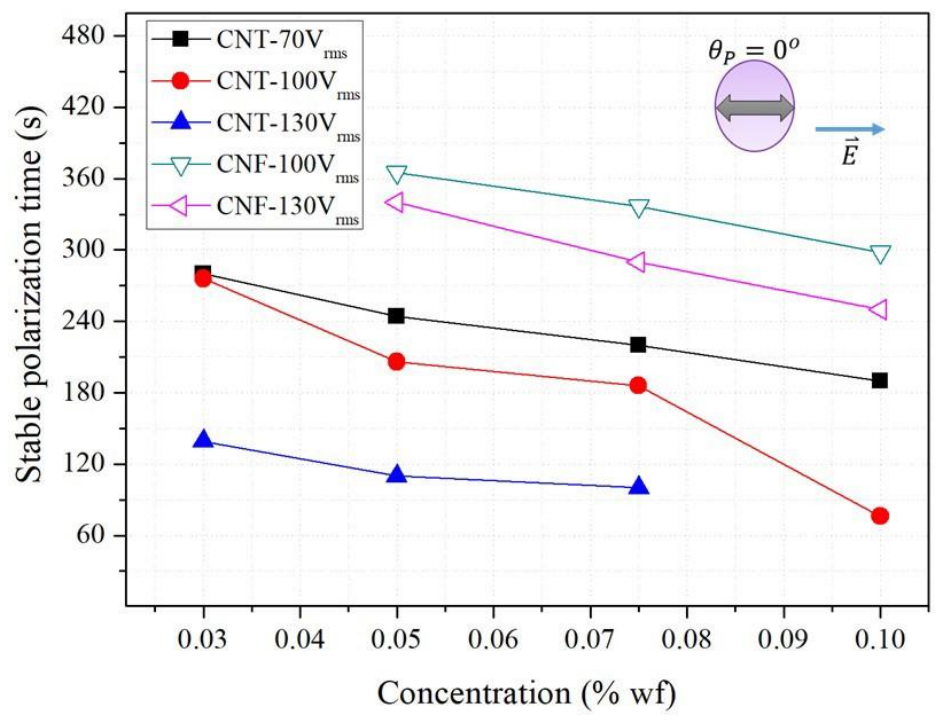

Figure 7 Stable polarization time when the incident light is polarized parallel to the chains from four different CNT (black, red and blue, solid symbol) and CNF (green, and pink, clear symbol) concentrations in ethylene glycol, when voltages of $70 \mathrm{~V}_{\text {rms }}$ (black), $100 \mathrm{~V}_{\text {rms }}$ (red and green) and $130 V_{\text {rms }}$ (blue and pink) were applied.

There are two interesting aspects to analyze from the obtained data. The first one is the time that it takes to the sample to stabilize and behave as a polarizer after turning on the voltage. In Figure 7, no polarizing effect is observed in CNF samples with $70 \mathrm{~V}_{\text {rms }}$ applied at any $\%$ wf concentration and neither the sample with $0.03 \%$ wf presents this effect at any of the applied voltages. Consequently, the chain structure is not dense enough to generate the effect. The CNT sample with $0.1 \%$ wf concentration and $130 \mathrm{~V}_{\text {rms }}$ applied was not tested because of the Joule effect, as has been mentioned before. In the CNT samples the period of time to stabilize always was shorter than those of CNF dispersions under the same conditions.

The second interesting aspect is how effective the samples are as polarizers, taking as reference the decrease in the magnitude of the transmitted light, which is presented in Figure 8 . The maximum achieved amplitude value was taken as $100 \%$. The samples with CNF show a similar behaviour regardless of the applied voltage, although a decrease in the amplitude is observed as the concentration of CNF increases. In samples with CNT, the decrease in amplitude is larger. Even at $0.075 \%$ wf the difference between the samples with CNF and CNT is evident. These differences are also a result of their 
difference in dispersibility as mentioned above. The behaviour of these materials opens the possibility of using them as tunable light modulators or as dynamic polarizers.

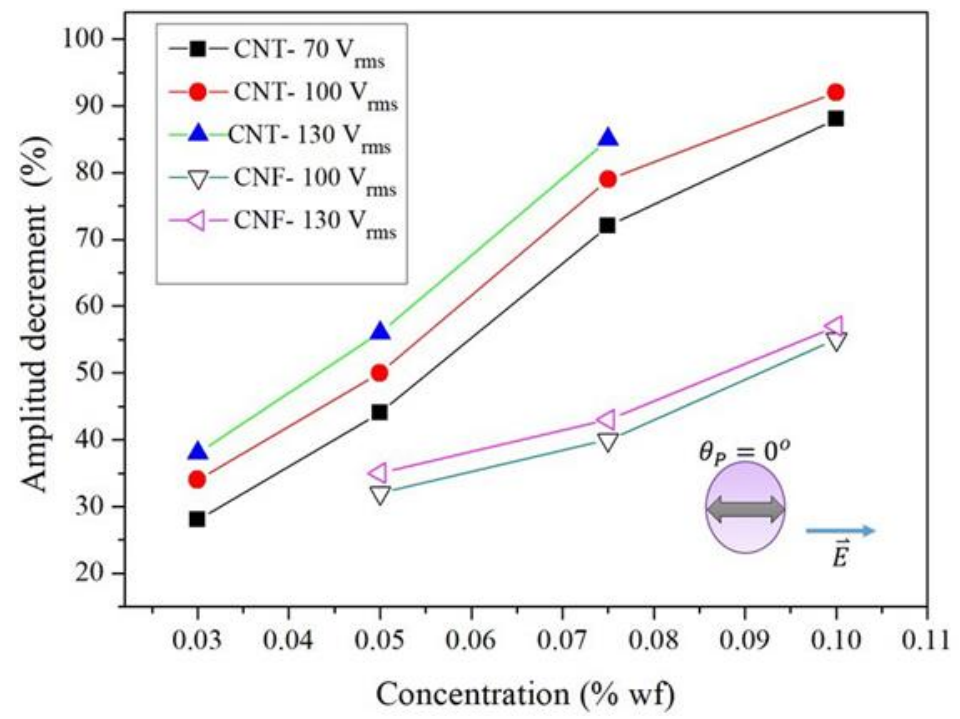

Figure 8 Amplitude percentage decrement when the polarized light is parallel to the chains from four different CNT (black, red and blue, solid symbol) and CNF (green, and pink, clear symbol) concentrations in ethylene glycol, when voltages of $70 V_{r m s}$ (black), $100 V_{r m s}$ (red and green) and $130 V_{r m s}($ blue and pink) were applied.

Based on the obtained results, it is interesting to determine the amount of light transmitted by the sample, when an electric field is applied, as a function of the angle of the polarization vector of the incident light. In Figure 9 the amplitude of polarized transmitted light is normalized to the amplitude of light transmitted when the polarized beam impinges, on the sample, perpendicular to the electric field generated by the applied 100

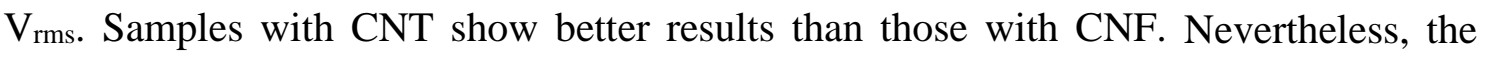
election of one or another kind of particles, besides the applied voltage, brings a wide range of possible applications. The sample with $0.1 \%$ wf achieves a light blocking effect of $95 \%$, compared to the amplitude when the beam is perpendicularly polarized with respect to when it is parallel, both with respect to the direction of the applied field. 


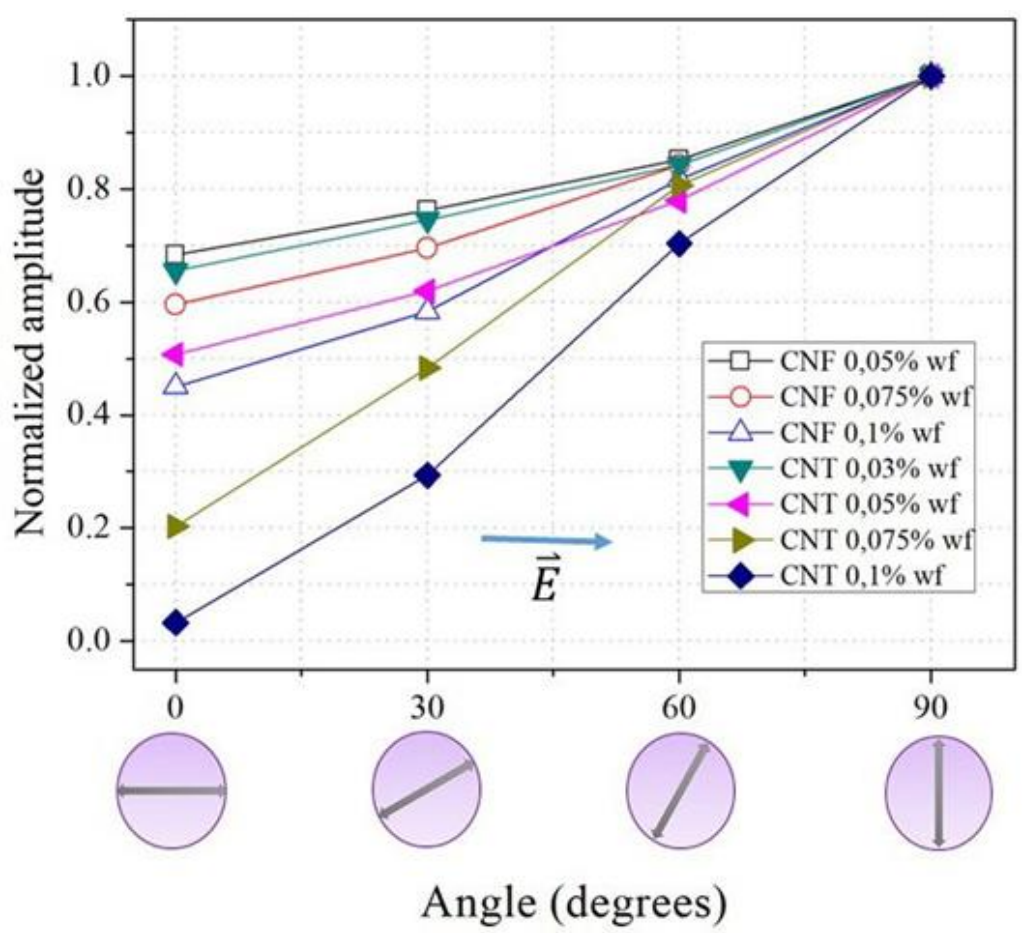

Figure 9 Amplitude response to angle rotation of the polarized light that impinges on the sample. Four different \% wf concentrations of CNT (green, pink, brown and violet, solid symbol) and $C N F$ (black, red and blue, clear symbol) were tested when a voltage $100 V_{\text {rms }}$ was applied.

In Figure 10, the measured amplitude for samples with nanoparticles dispersed in distilled water when the incident light is parallel to the chains structure is shown. Unlike the case of dispersions in ethylene glycol, the increase of the amplitude of the signal is not followed by a decay (see Figure 5). This happens because the chainlike structures are much more spaced, and the agglomeration of nanoparticles is greater due to their hydrophobic character. As a consequence, the dimensions of the gaps between the chains structure are larger, which prevents the polarizing effect. A sufficient amount of light pass through the distilled water without any interaction, although, this kind of samples can be used as mesh windows or light dimmers.

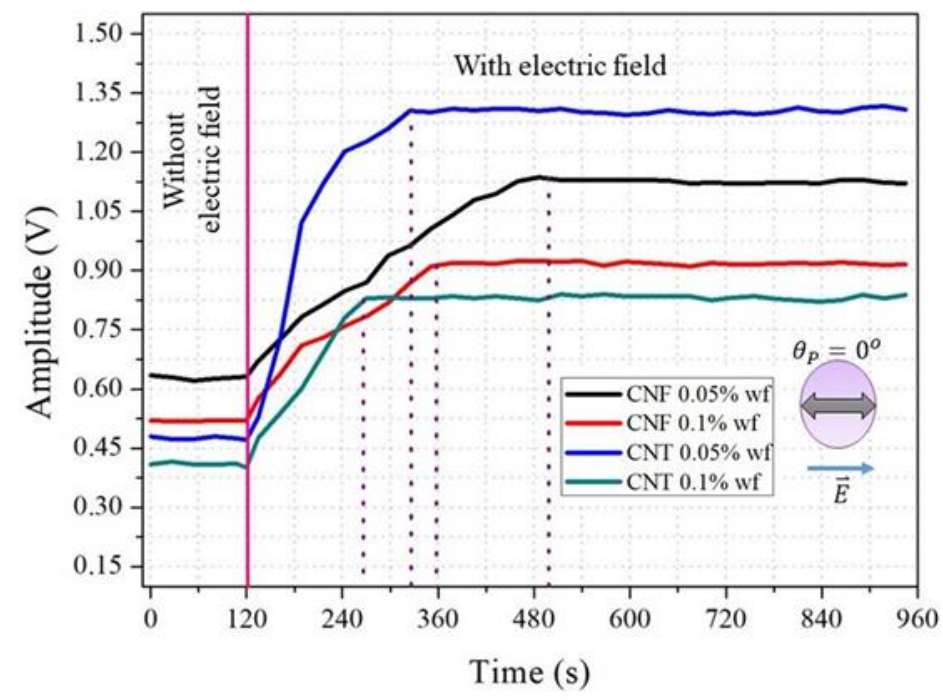

Figure 10 Evolution of the intensity of light polarized parallel to the chains direction, transmitted through dispersions of CNF (line black and red) and CNT(line blue and green) in distilled water, 
for two concentration $0.05 \% \mathrm{wf}$ (black and blue) and $0.1 \% \mathrm{wf}$ (red and green, from $120 \mathrm{~s}$ before switching on an ac voltage of $100 V_{r m s}$ during 800 seconds.

\section{Conclusions}

The application of an AC voltage to CNT and CNF ethylene glycol dispersions induces a concatenated structure of parallel chains of high particle concentrations. The time of alignment is inversely proportional to the concentration of nanoparticles and on the applied electric field intensity; both parameters increase the agglomeration and alignment rates. A polarizing effect appears due to the interaction of light with the chainlike nanoparticles structure. For all \% wf and applied voltage values, samples with CNT showed a better performance than those with CNF. This phenomenon is absent in the water dispersions due to the nanoparticles hydrophobic character, which prevents a proper structure formation.

The light transmission can be tuned varying the $\%$ wf, the applied electric field and the type of 1D carbon nanoparticles. The behavior of these composite fluids opens perspectives for the manufacturing of tunable light modulators, providing further development in manufacturing fast response and reversible dynamic polarizers, offering greater versatility than conventional polymer-based polarizers.

The simplicity with which these samples can be prepared make them ideal candidates to cover needs in practical applications, such as light dimmers in building windows and automotive industry. The results obtained open the possibility of manipulation of nanoparticles and the formation of structures with other types of interactions such as magnetic fields, to avoid the Joule effect inherent in the circulation of electric current.

\section{Acknowledgment}

This work has been partially supported by KU Leuven (C1-project OPTIPROBE--C14/16/063), SEP-CB-2015-01- 251882 and Project 98-SEP-Cinvestav Fund. APF-B acknowledges Conacyt for $\mathrm{PhD}$ scholarship as well as to "Becas Mixtas" fund for supporting their stay at the KU Leuven. Authors are grateful to Werner Neefs and Jose Bante Guerra for their technical support.

\section{References}

[1] Wang J, Chen Y and Blau W J 2009 Carbon nanotubes and nanotube composites for nonlinear optical devices J. Mater. Chem. 197425

[2] Bahr J L, Mickelson E T, Bronikowski M J, Smalley R E and Tour J M 2001

Dissolution of small diameter single-wall carbon nanotubes in organic solvents? Chem. Commun. 193-4

[3] Benedict X, Louie S G and Cohen M L 1995 Static Polarizabilities of single-walled carbon nanotubes Phys. Rev. B 52 8541-9

[4] Freitag M, Martin Y, Misewich J, Martel R and Avouris P 2003 Photoconductivity of single carbon nanotubes Nano Lett. 3 1067-71

[5] Stanway R 2004 Smart fluids: current and future developments Mater. Sci. Technol. 20 931-9

[6] Vivien L, Lançon P, Riehl D, Hache F and Anglaret E 2002 Carbon nanotubes for optical limiting Carbon N. Y. 40 1789-97

[7] Huang C W, Hsu C H, Kuo P L, Hsieh C Te and Teng H 2011 Mesoporous carbon 
spheres grafted with carbon nanofibers for high-rate electric double layer capacitors Carbon N. Y. 49 895-903

[8] Prasse T, Cavaillé J Y and Bauhofer W 2003 Electric anisotropy of carbon nanofibre/epoxy resin composites due to electric field induced alignment Compos. Sci. Technol. 63 1835-41

[9] Xiang J, Li J, Zhang X, Ye Q, Xu J and Shen X 2014 Magnetic carbon nanofibers containing uniformly dispersed $\mathrm{Fe} / \mathrm{Co} / \mathrm{Ni}$ nanoparticles as stable and high-performance electromagnetic wave absorbers J. Mater. Chem. A 2 16905-14

[10] Vales-Pinzón C, Alvarado-Gil J J, Medina-Esquivel R and Martínez-Torres P 2014 Polarized light transmission in ferrofluids loaded with carbon nanotubes in the presence of a uniform magnetic field J. Magn. Magn. Mater. 369 114-21

[11] Cervantes-Alvarez F, Macias J D and Alvarado-Gil J J 2018 Heat transport in electrically aligned multiwalled carbon nanotubes dispersed in water J. Phys. D. Appl. Phys. 51

[12] Brown M S, Shan J W, Lin C and Zimmermann F M 2007 Electrical polarizability of carbon nanotubes in liquid suspension Appl. Phys. Lett. 90

[13] Oliva-Avilés A I, Avilés F, Sosa V and Seidel G D 2014 Dielectrophoretic modeling of the dynamic carbon nanotube network formation in viscous media under alternating current electric fields Carbon N. Y. 69 342-54

[14] Bubke K, Gnewuch H, Hempstead M, Hammer J and Green M L H 1997 Optical anisotropy of dispersed carbon nanotubes induced by an electric field Appl. Phys. Lett. 71 1906-8

[15] Yamamoto K, Akita S and Nakayama Y 1996 Orientation of carbon nanotubes using electrophoresis Japanese J. Appl. Physics, Part 2 Lett. 35

[16] Yamamoto K, Akita S and Nakayama Y 1998 Orientation an durification of carbon nanotubes using ac electrophoresis J. Phys. D Appl. Phys. 31 L34

[17] Martin C A, Sandler J K W, Windle A H, Schwarz M K, Bauhofer W, Schulte K and Shaffer M S P 2005 Electric field-induced aligned multi-wall carbon nanotube networks in epoxy composites Polymer (Guildf). 46 877-86

[18] Zhu Y F, Ma C, Zhang W, Zhang R P, Koratkar N and Liang J 2009 Alignment of multiwalled carbon nanotubes in bulk epoxy composites via electric field J. Appl. Phys. 105

[19] Pech-May N W, Vales-Pinzón C, Vega-Flick A, Cifuentes Á, Oleaga A, Salazar A and Alvarado-Gil J J 2016 Study of the thermal properties of polyester composites loaded with oriented carbon nanofibers using the front-face flash method Polym. Test. 50 25561

[20] Vales-Pinzón C, Quiñones-Weiss G, Alvarado-Gil J J and Medina-Esquivel R A 2015 Heat Transport in Liquid Polyester Resin with Carbon Nanotubes Int. J. Thermophys. 36 2854-61

[21] Oliva-Avilés A I, Avilés F, Sosa V, Oliva A I and Gamboa F 2012 Dynamics of carbon nanotube alignment by electric fields Nanotechnology $\mathbf{2 3}$

[22] Murakami Y, Einarsson E, Edamura T and Maruyama S 2005 Polarization dependence of the optical absorption of single-walled carbon nanotubes Phys. Rev. Lett. 94 1-4

[23] Kumazawaa Y, Maniwaa Y, Umezub I, Ohtsukac Y, Achibac Y, Ohsawa I M- and Ohsawa I M- 1999 Optical Properties of Single-Wall Carbon Nanotubes Synth. Met. 103 $2555-8$

[24] Shoji S, Suzuki H, Zaccaria R P, Sekkat Z and Kawata S 2008 Optical polarizer made of uniaxially aligned short single-wall carbon nanotubes embedded in a polymer film Phys. Rev. B - Condens. Matter Mater. Phys. 77 1-4

[25] Kang B G, Lim Y J, Jeong K U, Lee K, Lee Y H and Lee S H 2010 A tunable carbon nanotube polarizer Nanotechnology 21

[26] Lynch M D and Patrick D L 2002 Organizing carbon nanotubes with liquid crystal solvents Nano Lett. 2 1197-201

[27] Jeong S H, Kim K K, Jeong S J, An K H, Lee S H and Lee Y H 2007 Optical absorption spectroscopy for determining carbon nanotube concentration in solution Synth. Met. 157 
$570-4$

[28] Jeong S J, Park K A, Jeong S H, Jeong H J, An K H, Nah C W, Pribat D, Lee S H and Lee Y H 2007 Electroactive superelongation of carbon nanotube aggregates in liquid crystal medium Nano Lett. 7 2178-82

[29] Reed B W and Sarikaya M 2001 Electronic properties of carbon nanotubes by transmission electron energy-loss spectroscopy Phys. Rev. B 64195404

[30] Yin J and Zhao X 2011 Electrorheology of nanofiber suspensions Nanoscale Res. Lett. 6 $1-17$

[31] Shao-Jie M and Wan-Lin G 2008 Mechanism of Carbon Nanotubes Aligning along Applied Electric Field Chinese Phys. Lett. 25 270-3

[32] Zhuo Chen, Yanlian Yang †, Fang Chen, Quan Qing, Zhongyun Wu and and Zhongfan Liu* 2005 Controllable Interconnection of Single-Walled Carbon Nanotubes under AC Electric Field J. Phys. Chem. B 109 11420-3

[33] Asthana R (Rajiv), Kumar A (Ashok) and Dahotre N B 2006 Materials processing and manufacturing science (Boston)

[34] Blatt S, Hennrich F, V. Löhneysen H, Kappes M M, Vijayaraghavan A and Krupke R 2007 Influence of structural and dielectric anisotropy on the dielectrophoresis of singlewalled carbon nanotubes Nano Lett. 7 1960-6 